\section{Regarding "18F-GP1, a Novel PET Tracer Designed for High-Sensitivity, Low-Background Detection of Thrombi”}

TO THE EDITOR: We read with great interest a recent publication by Lohrke et al. describing their preliminary data related to the detection of clots using ${ }^{18} \mathrm{~F}$-glycoprotein $1\left({ }^{18} \mathrm{~F}-\mathrm{GP} 1\right)$ as a PET tracer with high sensitivity for this purpose (1). The preliminary in vitro experiments demonstrated that ${ }^{18} \mathrm{~F}-\mathrm{GP} 1$ binds specifically with high affinity to the GPIIb/IIIa receptor, which is involved in platelet aggregation. In addition, these investigators generated successful results from cynomolgus monkey studies. The latter was accomplished by introducing catheters into the ascending aorta, carotid arteries, and vena cava. The authors correctly point out that previous attempts with radiotracer-based imaging to detect clots either in the venous or the arterial systems have had limited success for various reasons and that some have therefore been abandoned for this purpose. Overall, the presented results are interesting and merit further investigation, but we do have some comments and caveats.

Recently, we wrote 2 editorials describing the limitations of PET imaging in certain settings because of the physical and biologic shortcomings that are associated with the applications of this modality $(2,3)$. We have described several research initiatives that have not reached their intended aims despite promising results in animals and in vitro experiments. These limitations are also applicable to the detection of clots with PET radiotracers and should therefore be taken into consideration in future attempts to detect clots. Soon after the thrombosis occurs in an artery, it will be nearly impossible for radiolabeled tracers to reach the clot and allow its visualization by noninvasive imaging techniques. This is the case when clots are formed in critical arteries such as coronaries and tributaries of internal carotid arteries and when clots are formed in the pulmonary arteries after embolism from the peripheral veins. Investigators should be aware of this fact and be cautious when investing in research activities aimed at detecting clots in the arteries.

Furthermore, the overall mass of platelets that are incorporated into the thrombi may not provide the required volume and binding surface to be detected with in vivo PET if the agent clears rapidly from the circulation. Such agents may not prove to be suitable candidates for visualizing venous clots. Also, as we have pointed out in our recent editorials, the spatial resolution of PET imaging in human applications is approximately $8-10 \mathrm{~mm}$. As such, detection of small clots with PET may face serious challenges and not succeed in the future. The partial-volume effect, combined with rapid clearance of ${ }^{18} \mathrm{~F}-\mathrm{GP} 1$, may result in negative results, particularly with small clots. Thus, demonstrating the efficacy of the approach proposed by Lohrke et al. in practical and relevant clinical settings is crucial if the probability of success with such compounds is to remain high. In fact, similar attempts in the past with ${ }^{99 \mathrm{~m}} \mathrm{Tc}$-apcitide have shown suboptimal results (4).

However, it is feasible to detect clots in the venous system that only partially occlude the lumen of the affected vessel, as administered radiotracers can reach and label the targeted cells (platelets or white cells) or other biologic sites (fibrin). This labeling can result in visualization of the venous clots with relatively high success. Attempts to visualize clots with antifibrin antibodies were successful based on past experience $(5,6)$. Sim- ilarly, targeting activated inflammatory cells such as white cells that are incorporated into the clot results in detection of venous thrombi by ${ }^{18} \mathrm{~F}$-FDG PET imaging. In fact, preliminary published data indicate that the sensitivity of ${ }^{18} \mathrm{~F}-\mathrm{FDG}$ in detecting venous clots is nearly $100 \%(7-9)$. ${ }^{18} \mathrm{~F}-\mathrm{FDG}$ and radiolabeled antibodies remain in the circulation for an extended period, allowing significant accumulation of the tracer at the thrombotic sites over time.

We would like to draw attention to ${ }^{18} \mathrm{~F}-\mathrm{FDG}$ results previously presented by our group and others, as they are similar to those presented by Lohrke et al. Several of the (very relevant) questions and caveats put forth by Lohrke et al. in their introduction are already assessable by ${ }^{18} \mathrm{~F}$-FDG, and some of the limitations of ${ }^{18} \mathrm{~F}$ FDG are likely also true for ${ }^{18} \mathrm{~F}-\mathrm{GP} 1$. First, only very acute venous (and arterial) nonoccluding thrombi were assessable with ${ }^{18} \mathrm{~F}-\mathrm{GP} 1$ in the setup presented, but it remains unknown how the uptake is in older, chronic thrombi. This question has already been successfully evaluated using ${ }^{18} \mathrm{~F}-\mathrm{FDG}$, with clear signs of declining ${ }^{18} \mathrm{~F}$ FDG avidity over time $(10,11)$. Also, the catheter-based method favors thrombus formation in the venous bed and major arteries, but there is no evidence of any ${ }^{18} \mathrm{~F}-\mathrm{GP} 1$ uptake related to pulmonary embolism - a major drawback of ${ }^{18}$ F-FDG $(9,12)$. Finally, it is perceivable that ${ }^{18} \mathrm{~F}-\mathrm{GP} 1$ better discriminates conventional bland thrombi from etiologies such as septic emboli, but this discrimination still requires prior imaging with sufficient sensitivity to detect the thrombus in the first place, and ${ }^{18} \mathrm{~F}-\mathrm{FDG}$ has demonstrated high sensitivity for several etiologies, including conventional bland thrombi, septic emboli, and tumor thrombosis $(8,9,13)$.

We believe the future management of patients with thrombotic disorders will heavily depend on the detection and characterization of clots in the venous system, and despite the interesting preliminary results presented by Lohrke et al., ${ }^{18}$ F-FDG PET currently has the greatest potential for this purpose. If validated by well-designed prospective clinical trials, ${ }^{18} \mathrm{~F}-\mathrm{FDG}$ PET imaging has great potential for replacing or at least complementing other diagnostic modalities for accurate diagnosis of thromboembolic disorders.

\section{REFERENCES}

1. Lohrke J, Siebeneicher H, Berger M, et al. ${ }^{18} \mathrm{~F}-\mathrm{GP} 1$, a novel PET tracer designed for high-sensitivity, low-background detection of thrombi. $\mathrm{J} \mathrm{Nucl}$ Med. 2017;58:1094-1099.

2. Alavi A, Werner TJ, Hoilund-Carlsen PF. What can be and what cannot be accomplished with PET to detect and characterize atherosclerotic plaques. J Nucl Cardiol. July 10, 2017 [Epub ahead of print].

3. Alavi A, Werner TJ, Hoilund-Carlsen PF. What can be and what cannot be accomplished with PET: rectifying ongoing misconceptions. Clin Nucl Med. 2017;42:603-605

4. Taillefer R, Edell S, Innes G, Lister-James J. Acute thromboscintigraphy with ${ }^{99 \mathrm{~m}} \mathrm{Tc}$-apcitide: results of the phase 3 multicenter clinical trial comparing ${ }^{99 \mathrm{~m}} \mathrm{Tc}$-apcitide scintigraphy with contrast venography for imaging acute DVT. Multicenter Trial Investigators. J Nucl Med. 2000;41:1214-1223.

5. Alavi A, Palevsky HI, Gupta N, et al. Radiolabeled antifibrin antibody in the detection of venous thrombosis: preliminary results. Radiology. 1990;175:7985 .

6. Schaible TF, Alavi A. Antifibrin scintigraphy in the diagnostic evaluation of acute deep venous thrombosis. Semin Nucl Med. 1991;21:313-324.

7. Houshmand S, Salavati A, Hess S, Ravina M, Alavi A. The role of molecular imaging in diagnosis of deep vein thrombosis. Am J Nucl Med Mol Imaging. 2014;4:406-425.

8. Ravina M, Hess S, Chauhan MS, Jacob MJ, Alavi A. Tumor thrombus: ancillary findings on FDG PET/CT in an oncologic population. Clin Nucl Med. 2014;39:767-771. 
9. Hess S, Madsen PH, Iversen ED, Frifelt JJ, Hoilund-Carlsen PF, Alavi A. Efficacy of FDG PET/CT imaging for venous thromboembolic disorders: preliminary results from a prospective, observational pilot study. Clin $\mathrm{Nucl}$ Med. 2015;40:e23-e26.

10. Rondina MT, Lam UT, Pendleton RC, et al. ${ }^{18} \mathrm{~F}-\mathrm{FDG}$ PET in the evaluation of acuity of deep vein thrombosis. Clin Nucl Med. 2012;37:1139-1145.

11. Zhu HJ, Hess S, Rubello D, Goris ML, Alavi A. The strong but nonspecific relationship between ${ }^{18} \mathrm{~F}$-FDG uptake in the lower-extremity veins and venous thromboembolism. Nucl Med Commun. 2016;37:322-328.

12. Flavell RR, Behr SC, Brunsing RL, Naeger DM, Pampaloni MH. The incidence of pulmonary embolism and associated FDG-PET findings in IV contrastenhanced PET/CT. Acad Radiol. 2014;21:718-725.

13. Nielsen AL, Thomassen A, Hess S, Alavi A, Hoilund-Carlsen PF. Deep venous thrombosis and pulmonary embolism detected by FDG PET/CT in a patient with bacteremia. Clin Nucl Med. 2013;38:276-277.

\section{Abass Alavi* \\ Thomas J. Werner Søren Hess \\ Poul Flemming Høilund-Carlsen *Hospital of the University of Pennsylvania 3400 Spruce St. Philadelphia, PA 19104. \\ E-mail:abass.alavi@uphs.upenn.edu}

Published online Sep. 14, 2017.

DOI: 10.2967/jnumed.117.200378

REPLY: In our recently published article, we describe the performance of a new tracer, ${ }^{18} \mathrm{~F}-\mathrm{GP} 1$, in a well-established extracorporeal human blood circuit and in a nonhuman primate model (1). We are fully aware of limitations imposed by any model and would like to point to a phase 1 clinical study that is currently ongoing to investigate ${ }^{18} \mathrm{~F}-\mathrm{GP} 1$ in subjects with acute symptoms of deep vein thrombosis and pulmonary embolism (NCT02864810). Preliminary interim data from this study that were presented recently at the SNMMI 2017 annual meeting confirm the preclinical data on ${ }^{18} \mathrm{~F}-\mathrm{GP} 1$ biodistribution and its ability to image thrombi in humans (2). Of note, thromboembolic foci have been successfully detected in all patients enrolled with acute pulmonary embolism and deep vein thrombosis so far, and the coincident occurrence of deep vein thrombosis and pulmonary embolism was common, as would be expected. Although the clinical study results are preliminary and a manuscript has not yet been peer-reviewed, they show that ${ }^{18} \mathrm{~F}-\mathrm{GP} 1$ behaves analogously in humans and nonhuman primates. We can only surmise that there are enough activated platelets in venous clots to generate a robust signal given the high affinity and selectivity of the tracer.
Dr. Alavi et al. reported on the potential use of ${ }^{18} \mathrm{~F}-\mathrm{FDG}$ in the assessment of deep vein thrombosis if the results from a small study are confirmed in a larger prospective trial (3). However, we have doubts of the general utility of ${ }^{18} \mathrm{~F}-\mathrm{FDG}$ in clinical routine. Because ${ }^{18} \mathrm{~F}-\mathrm{FDG}$ visualizes only thrombus-associated inflammation, it can be only a surrogate marker of thrombosis. ${ }^{18} \mathrm{~F}-\mathrm{FDG}$ uptake is not representing a critical mechanism in clot formation. We believe that accurate detection of the thrombus itself, without the associated inflammatory processes, is critical to a successful thrombus tracer. Nonspecificity may limit the use of ${ }^{18} \mathrm{~F}-\mathrm{FDG}$, particularly with regard to monitoring of targeted pharmacologic intervention. In contrast, the ability to track activated platelets by a specific targeted PET probe would allow antithrombotic therapies to be optimized through titration of antithrombotic and antiplatelet agents.

Many nuclear medicine tracers over the years have not performed adequately in the detection of pulmonary emboli. The inability of a tracer to access the emboli, the small size of the emboli, or the loss of the tracer target from the emboli are possible reasons. It is hoped that in the future a tracer that is well characterized and validated will be able to help address these possibilities.

Although there is still much to learn about ${ }^{18} \mathrm{~F}$-GP1, the discussed preclinical article, bolstered by early clinical data, indicates that ${ }^{18} \mathrm{~F}-\mathrm{GP} 1$ is a promising tracer, especially with respect to the high signal-to-noise ratio and the high specificity for GPIIb/IIIa on platelets over the many other integrins involved in inflammation and endothelial activation. We eagerly await additional clinical data.

\section{REFERENCES}

1. Lohrke J, Siebeneicher H, Berger M, et al. ${ }^{18} \mathrm{~F}-\mathrm{GP} 1$, a novel PET tracer designed for high-sensitivity, low-background detection of thrombi. J Nucl Med. 2017;58:10941099.

2. Jin S, Lee JS, Han Y, et al. Exploratory clinical trial of $\left[{ }^{18} \mathrm{~F}\right] \mathrm{GP} 1$ for imaging arterial or venous thromboembolism using positron emission tomography: interim analysis of an open-label, single center study [abstract]. J Nucl Med. 2017;58(suppl 1):438.

3. Hess S, Madsen PH, Iversen ED, Frifelt JJ, Hoilund-Carlsen PF, Alavi A. Efficacy of FDG PET/CT imaging for venous thromboembolic disorders: preliminary results from a prospective, observational pilot study. Clin Nucl Med. 2015;40:e23-e26.

\author{
Andrew Stephens \\ Piramal Imaging $\mathrm{GmbH}$ \\ Tegeler Strasse 6/7 \\ Berlin, 13353, Germany \\ E-mail: andrew.stephens@piramal.com
}

Published online Oct. 30, 2017.

DOI: 10.2967/jnumed.117.203687 